\title{
Short review on the aggressive behaviour: genetical, biological aspects and oxytocin relevance
}

\author{
PADURARIU Manuela ${ }^{1}$, PREPELITA Raluca ${ }^{1}$, CIOBICA Alin ${ }^{2,3}$, \\ DOBRIN Romeo ${ }^{1}$, TIMOFTE Daniel $^{1 *}$, STEFANESCU Cristinel ${ }^{1}$, \\ CHIRITA Roxana ${ }^{1}$
}

\author{
14 "Gr. T. Popa" University of Medicine and Pharmacy, lasi, Romania \\ 2 "Alexandru loan Cuza" University, lasi, Romania \\ ${ }^{3}$ Center of Biomedical Research of the Romanian Academy, lasi, Romania \\ *dantimofte@yahoo.com
}

Keywords: aggressive behaviour, genetics, biological mechanisms, oxytocin.

\begin{abstract}
In this mini-review we were interested in describing the main genetic, biological and mechanistic aspects of the aggressive behaviour in human patients and animal models. It seems that violent behaviour and impulsive traits present a multifactorial substrate, which is determined by genetic and non-genetic factors. Thus, aggressivity is regulated by brain regions such as the amygdala, which controls neural circuits for triggering defensive, aggressive or avoidant behaviour. Moreover, other brain structures such as the anterior cingulate cortex and prefrontal cortex regions could modulate circuits involved in aggression. Regarding the genetic aspects, we could mention the mutations in the monoamine oxidase or the polymorphisms of the genes involved in the metabolism of serotonin, such as tryptophan hydroxylase. Also, besides the low levels of serotonin metabolites, which seem to be associated with impulsive and aggressive traits, there are good evidences that deficiencies in glutamate transmission, as well as testosterone, vasopressin, hypochloesterolemia or oxytocin modifications could be related to the aggressive behaviour. Regarding oxytocin we present here in the last chapter the controversial results from the current literature regarding the various effects exhibited by oxytocin administration on the aggressive behavior, considering the increased interest in understanding the role of oxytocin on the main neuropsychiatric disorders.
\end{abstract}

\section{Introducing the concept of aggression}

Based on the etymological analysis, the word aggression comes from Latin, from aggressio, signifying attack, and also from the verb aggredior, which can be translated as "to go against something or someone", "to approach someone with a purpose ", or "to assault" [1]. Thus, aggression basically describes a state of the psychophysiological system that allows the individual to respond with a set of hostile behaviours in the conscious, unconscious, or phantasy plan towards the destruction, coercion, degradation, humiliation of a meaningful thing or being, that the abuser perceives as such and represent a challenge for him/her [2].

Moreover, according to a general dictionary of psychology, the term aggression is defined as: 1 . the tendency to display hostility by manifestation of aggressive acts; 2 . the tendency to overcome any encountered oppositions; 3. the tendency of self-assertion by unswerving manifesting of self interests; 4. hiperenergy in one's attitudes and reactions; 5. the constant tendency of domination within the social group or community [3].

In addition, from a psycho-sociological perspective, the aggressiveness as physical expression of the aggressive impulses is perceived by the existence of the behavioural intent to cause moral or psychological suffering to others. The human aggressive behaviour has on one hand an innate feature and, on the other hand, is acquired during the course of anthropogenic evolution. Also, if in the primitive societies the aggression consisted only in physical attacks as violence, in modern society the aggressive acts and deeds are sometimes indirect manifestations of aggression. 
Moreover, the ethological vision on the concept of aggression is also interesting. From this perspective, aggression refers to the interaction and evolution of animals in natural environments. The ethologists analyze human aggression from the perspective of animal aggression. Thus, biologically, people are a highly evolved animal species. In the animal world aggression includes three functions: equal distribution of the species, the selection of the fittest and the protection of the young and defenceless [4].

In such cases in animals, aggression can involve physical violence, hitting, biting or pushing, and most conflicts are resolved through menacing and intimidation stances or strokes that do not cause physical damage. The stereotype signals may include threatening and hostile facial expressions; vocalizations, such as birdsongs and the release of chemicals [5]. In animals, aggressive behaviour might confer biological advantages. Moreover, aggression can ensure gaining territories, resources of food and water, provides opportunities for mating, for self-defense or protection of offspring and leads to the natural selection of more vigorous animals [6]. Also, an individual from a group is more likely to become aggressive when the other members exhibit aggressive behaviours that are similar [7].

In analogy with the aggressive behaviour in animals, the human aggressiveness retains an important role in the survival instinct and exhibits. Still, a variety of the roles of the aggressive behaviour are no longer valid today. Also, people can turn aggressive energy by sublimating it into work, play, sports competition or art. Given the specific energy of this pulsing, aggression seems to be unavoidable, as it may manifest spontaneously, regardless of situational particularities. Aside from its obvious internal nature, aggression can be decreased or maintained by the course of development in the context of culture and environment $[8,9]$.

In this way, aggression is a characteristic of all living beings throughout their evolutionary scale, having its origin in the central nervous system primitive structures. Moreover, it is a form of destructive behaviour intended to cause damage, either material, psychological, moral or mixed. Thus, the act of aggression may be directed against objects such as a house, furniture, kitchen utensils, against human beings or against one's ownself as autolytic behaviour. Thus, aggression can take several forms: aggression against other persons is referred to as hetero-aggression, which can be physical or verbal. The milder variant of this form of aggression is hostility and the extreme variant is murder. Classic aggression is directed against objects and things from the environment and is aimed to destroy the objects, while directing the autolytic behaviour against one's ownself is called self-aggressiveness, presenting a broader spectrum of manifestations, from self-hostility and self-sabotage to self-mutilation and reaching suicidal tendencies and/or completed suicide. When it occurs as an impulsive reaction, the manifestation of aggressiveness has the role to release the tension, to psychologically defuse an individual with an excessively high psychoenergetic burden. In some cases, the planned hetero-aggressiveness can indicate the presence of psychopathic traits of personality and self-aggression could be an indicator of depression [10].

Also, certain sources of aggression are related to the personality traits of the individual, while others to external conditions, such as frustration, which is one of the most common triggers of aggression along with the attack or direct provocation, verbal and physical, pain, in its physical and moral forms, heat and crowds. The most common and well-known forms of aggressive behaviour that have a social and community impact are particularly delinquency and crime. Thus, aggression is a behaviour oriented to produce damage, injuries, prejudice and physical harm to objects, people or one's own self. Also, aggressiveness can be manifested in different forms and intensities, from simple ideas and thoughts, physical arousal, anger, competitive type traits and dominance to verbal aggression and serious violence acts.

In this way, the researches on adult aggressive behaviour demonstrated two types of aggression: proactive aggression usually calculated and making use of tools, aimed to obtain rewards and reactive aggression, which is generally impulsive as a stress adaptation response for unexpected events and can be a potential hazard [11]. 


\section{Epidemiology}

There are relatively few studies in the literature regarding the general aggressive behaviour. In this way, in a study from 2000 performed on 1269 patients with various psychiatric disorders, an overall rate for aggressive behaviour of $13.7 \%$ was reported. The highest rates in terms of aggressive behaviour occurred more often in patients with bipolar disorders $(2.81 \%)$ and schizophrenia (1.96\%). Moreover, the patients at increased risk were those under 32, with episodes of psychosis or substance abuse [12].

Regarding the way people manifest aggressive behaviour, it is known that this specific behaviour is exhibited in various degrees in individuals varying by gender, age, cultural aspects, as well as by biological and genetic peculiarities or presence of certain affections.

Thus, in children there is an almost constant degree of aggressiveness, manifested either as a healthy assertive, competitive behaviour or as a pathological trait frequently involving violent behaviour, delinquency and criminality. Also, it is known that in boys and males in general, the level of aggressivity is higher than in women and is primarily geared towards persons of the same age. Also, the predisposing precipitating factors for aggressive behaviour in children are different, depending on their age. Thus, in little children the lack of attention and physical discomfort can be causes of violent explosions of anger. Later, insults, criticisms or social comparison are triggers for aggressive behaviour, while in adolescents, the frustrations may be hidden under a rather masked form as breaking rules, stealing, lying, cheating or the need for social dominance [13].

\section{Genetical aspects}

The genetic substrate has also a particularly important role in the expression of aggressive behaviour and in the presence or absence of the personality traits associated with aggression. In this way, the studies on twins or adoptions suggested that heredity is involved in aggresivity, in varying proportions (e.g. from $44 \%$ to $72 \%$ ) [14].

However, not a single gene has been identified as to be clearly associated with this type of behaviour, but rather a polygenetic substrate formed from multiple genes that regulate the activity of some neurotransmitters such as serotonin or genes responsible for the structural components of brain areas critical for aggression. Moreover, this genetic polymorphism may contribute to individual differences and susceptibility to aggressive behaviour. Thus, the mutations in the monoamine oxidase (MAO) gene which are associated with the alteration of catecholamines metabolism, or polymorphisms of the genes involved in the metabolism of serotonin, such as tryptophan hydroxylase of the 5-HT1B, the 5-HT2A and 5-HT1A receptor have been identified [15]. Also, one allele of the tryptophan hydroxylase gene was associated with the suicide attempts of violent delinquents and with aggressive behaviour in some patients with personality disorders [16].

Also, the genetic predisposition for aggressivity appears to be deeply affected by genetic polymorphic variants of the serotonergic system affecting the level of serotonin in the central and peripheral nervous system, the biological effects of this hormone, the serotonin production rate, the synaptic release and degradation. In this way, some functional polymorphisms of monoamine oxidase A (MAOA) and the serotonin transporter (5-HTT) are of particular importance considering the connections between the aformentioned polymorphic variants and anatomical changes in the limbic system of aggressive persons. Furthermore, the functional variants of the 5-HTT and MAOA genes can intervene in how the environmental factors influence the aggressive traits [17].

\section{Biological mechanisms for aggressiveness}

The neurobiological bases of aggressive behaviour consist from a complex of molecules and neural circuitry designed to convert motivation into action. Thus, the exposure to various frustrating stimuli such as abuse, frustration or hostility can stimulate certain brain region that process emotional and cognitive stimuli and increase psychic excitability. Moreover, it has been shown that 
impulsiveness and violence are associated with specific brain regions, such as the limbic system. In this way, brain structures which are considered essential in triggering aggressive behaviour are represented by the amygdala, the ventromedial hypothalamus, the limbic system, the motor cortex and orbitofrontal cortex [18]. Moreover, in patients with dementia the level of agitation and aggression are directly proportional to the level of atrophy in certain brain key areas for aggressive behaviour, such as the frontal lobe, amygdala, cingulate gyrus or the hippocampus. Also, the amygdala responds to threats and provocative stimuli by stimulating the motor cortex which further initiates the motor component of the aggressive act [18].

The emotional component is also associated with the cingulate cortex, which analyzes the negative emotional stimuli. Additionally, the amygdala has connections with the hippocampus and is involved in releasing certain factors that have the role of changing the homeostasis of the body (e.g. as to prepare it for action). Also, the system limiting the aggressive behaviour has its origin in the prefrontal cortex and in particular in the orbital prefrontal cortex, inhibiting the limbic regions involved in the generation of the aggressive behaviour [19]. Moreover, in experimental animals, stimulating ventromedial hypothalamus causes aggressivity and inhibits the natural structures responsible for natural aggressivity inhibition [20].

Further evidence is also provided by studies of borderline personality disorder manifested through aggressive behaviour, impulsivity, physical aggression directed towards others, acts of selfmutilation, and family violence, showing changes in the serotonergic system of these patients. Also, a link between temporal lobe epilepsy and violent and impulsive behaviour was described, and an association between aggressive behaviour in patients who have a history of head injury and brain organic changes [21].

Of course, the way in which aggressive behaviour is expressed is also based on the various specific neurotransmitter systems. In this way, the most studied system involved in aggression is the serotonin system. In this way, a decrease in the serotonergic transmission, which can be induced by the inhibition of serotonin production or by antagonizing its effects, determines a decrease of the negative consequences or of the relevance of punishment for a certain type of behaviour. Thus, restoring serotonin through the administration of L-tryptophan (e.g. a serotonin precursor) or drugs that increase serotonin level could strength the behavioural effects of punishment. Moreover, the restoration of serotonergic activity by administration of L-tryptophan or drugs that increase serotonin could perhaps help recover control over violent tendencies [22].

Also, as serotonin seems to facilitate the inhibition of prefrontal cortex, insufficient serotonergic activity can lead to increased aggression. The decrease of serotonin levels, as demonstrated by identifying low levels of a serotonine metabolite, has been associated with impulsiveness and violent behaviour. In this way, studies on the serotonergic neurotransmitter system show that serotonin metabolite, 5-hydroxyindoleacetic acid (5-HIAA) is found in low concentrations in the cerebrospinal fluid in depression and could be accompanied by violence and suicidal behaviour [23].

Moreover, other authors demonstrated that there is a correlation between the level of 5hydroxyindoleacetic from the cerebrospinal fluid and impulsive and violent behaviour. Thus, a low concentration of the serotonin metabolite is found in people with aggressive behaviour. It also seems that a low level of 5-HIAA is present in delinquents or people with a history of violence [24].

In addition, it is believed that low levels of serotonin metabolites seem to be associated with impulsive and aggressive traits. As mentioned before, it seems that serotonin depletion is associated with increased aggressiveness and impulsivity. In this way, a 2013 study on transgenic mice showed that a chronic reduction in the levels of serotonin is associated with increased aggresivity. Moreover, pharmacological intervention on serotonergic neurons, aimed to suppress the neurotransmitter discharge, resulted in increased levels of aggression [25]. These data confirmed the fact that low serotonin activity is decreasing the threshold for aggressive behaviour and supports the idea of a direct association between low serotonin levels and increased aggressiveness.

Another relevant aspect in this matter could be represented by some childhood experiences such as trauma or abuse, in relation to the emergence of serotonergic system abnormalities. In this way, 
some studies showed that sexually abused women are experiencing genetic changes associated with a low activity of monoamine oxidase A allele, a gene involved in serotonin synthesis [26]. Moreover, these women have subsequently increased rates of antisocial traits. Thus, changes in the serotonin system may be actively contributing to strengthening hostile, aggressive and impulsive personality traits, especially when exposed to negative experiences [26].

Also some researchers reported an interaction between genetic, environmental and gender factors, especially during the critical early stages of development, which causes pathological manifestations that reflect changes in serotonin homeostasis. Additionally, the serotonin system involvement in aggressive behaviour could be the outcome of various homeostatic imbalances for the 5-HT system [27].

Also, some clinical studies suggested that the increased reactivity of noradrenergic and dopaminergic system may facilitate aggression. Thus, reduced levels of norepinephrine may be responsible for triggering excessive irritability in response to a stressful, unpredictable factor. Biological, biochemical and genetic investigations of the enzyme responsible for the metabolism of catecholamines, the MAO-A, have also shown that low levels of MAO-A activity are associated with susceptibility to react violently and with impulsive behaviour [28,29]. Moreover, it appears that in males the antisocial characteristics are negatively correlated with the activity of MAO [30].

In addition, the involvement of glutamate in aggressive behaviour has been investigated in several studies, as some theoretical models indirectly link impulsivity and aggression to glutamate. As know, glutamate is the most abundant excitatory neurotransmitter in the vertebrate nervous system and is released from presynaptic vesicles after stimulation of the presynaptic neurons, acting on specific receptors, the N-methyl-D-aspartate (NMDA) receptor, and $\alpha$-amino-3-hydroxy-5methyl-4-isoxazolepropionic acid (AMPA) [31]. Also, beside the preclinical studies which are suggesting that central glutamate receptors stimulation could increase the aggressive behaviour [32], in some experimental animals the administration of glutamate directly into the central gray matter induces defensive hostility, while the treatment with a glutamate antagonist such as the kynurenic acid results in the same response, of even defensive aggressiveness [33]. In fact, there is also a considerable number of glutamatergic neurons within the projections between the anteromedial hypothalamus and central gray matter, which could represent the structural support for the link between glutamate and aggressiveness [32]. These data are in fact supported by a study published in 2013 showing that there is a positive correlation between the CSF glutamate levels and levels of impulsive aggressivity in human patients [34].

Regarding the endocrine system involvement in the aggressive behaviour, it is believed also that testosterone and mainly its most active metabolite dehydroepiandrostenedione (DHEA) could be implicated. Thus, it was showed that testosterone levels are higher in people with aggressive behaviour, as in the case of the convicts which have committed violent crimes [35]. Also, high levels of testosterone occur in sport teams that have an aggressive, dominant component or in various confrontations [36]. The testosterone acts centrally through the activation of amygdala for example, triggering aggressivity, while peripherally it increase muscle mass to achieve specific motor behaviour. Also, a large number of receptors for androgens and estradiol are found in the neurons from the prefrontal area, hypothalamus and especially the amygdala. Moreover, the effect of testosterone on the brain begins in early embryonic life, leading to anatomic and organizational changes that produce, in fact, the masculinization of the brain. Also, the antiandrogenic agents appear to reduce the level of aggressivity [37].

We should also mention in this context the relevance of the hypothalamic-pituitary-adrenal (HPA) axis, as well as the importance of cortisol and their relations to serotonergic system, which antagonize the effects of testosterone [38,39]. Moreover, it seems that a major role in the increase or decrease of the impulsivity is played by the imbalances between testosterone and serotonin or testosterone and cortisol (e.g. high levels of testosterone and low concentrations of cortisol), which is explicable considering the reduced activity of the control and emotion self-regulating neural circuitry. 
Moreover, hypochloesterolemia has been associated with aggressive behaviour and aggressive suicidal attempts, while lipid-decreasing drugs administration correlates with increased irritability states [40]. Also, experiments on primates have shown that reducing cholesterol through diet could actually lead to increased aggressiveness and reduced activity for the central serotonin, which is associated with the risk of violence in both humans and animals [41].

In addition, there are also studies demonstrating that vasopressin increases aggression behaviour in most mammals including humans. It appears that this effect could be linked to the aforementioned serotonin system effects, manifested through a modulating effect on the aggressive behaviour [42]. Also, some data supports the correlation between the level of CSF vasopressin and a personal history of aggressivity [43]. As already mentioned, vasopressin exerts uninhibitory actions via the serotonin system, aspects which is somehow supported by the fact that vasopressin antagonists can reduce aggressive behaviour [44].

\section{Oxytocin and aggression}

Another peptide related to vasopressin is oxytocin (e.g. the vasopressin/oxytocin signalling systems are involved in a variety of functions such as reproduction, immunity and thermoregulation, but with focus on the social manifestations connected with affiliation and aggression [42]) and lately, there is increased interest in understanding the role of (especially intranasal) oxytocin on the main neuropsychiatric disorders such as autism [45], schizophrenia [46], anxiety [47], depression, Prader Willi syndrome [48] or even psychopathy [49] and the variety of behaviours exhibited by the relevant central areas, including aggression [50-52].

In this way, it was demonstrated several times, by authors such as Bosch et al., in 2005 that oxytocin is critically involved in the regulation of maternal aggression [53]. Moreover, the aforementioned author did manage to find a very significant correlation between the aggressive behaviour showed as a part of maternal defence in specific behavioural testing and oxytocin release from both the paraventricular nucleus and the central nucleus of the amygdala. Also, mechanistically speaking it seems that the most important aspect in oxytocin modulation for the maternal aggressive behaviour is represented by the differences in the central release patterns of oxytocin [53]. Moreover, in a subsequent publication in 2013 the same group is stressing the aforementioned connection between oxytocin and arginine vasopressin in modulating maternal aggression in rats, developing also a further hypothesis on the commune role of these neuropeptides in anxiety perception and how this can be correlated to maternal aggression [54], considering also the recent reports regarding the significant effects of intransal oxytocin administration in anxiety patients [47].

Interestingly, it was also recently showed that in the high trait aggressive people the administration of oxytocin can result in an increased aggression towards a close person (e.g. intimate or romantic partner), possibility as a way of maintaining the current status/relationship [55]. In this way, in 2014 the group of Nathan DeWall showed in a in a double-blind, placebocontrolled study of high trait aggressive subjects that oxytocin (24 International Units) is actually stimulating aggression only in subjects prone to physical aggression (for example exhibiting behaviours such as hitting or throwing objects etc.) [55].

Similar aspects were also showed in various other experimental species such as dogs, fish or piglets. In this way, the Topal group showed very recently in 2015 that dogs receiving intranasal oxytocin showed less friendly first reaction and individual differences in aggression to an unfamiliar experimenter, as compared to placebo, in a specific design behavioural task for dogs called Threatening Approach Test [50].

Also, in piglets, which are considered to be much more closer in brain anatomy, growth and development to the human brain, as compared for example to the classical rodent models [56], the group of Rault et al. in Australia showed that neonatally oxytocin-treated piglets received and performed more aggressive behaviours, then the controls, possibly by reducing the HPA axis [51].

Moreover, in fish such as Neolamprologus pulcher, a cooperatively breeding cichlid fish, Hellmann group showed also in 2015 , that after temporarily removing a subordinate individual, it 
was more likely for this to receive increased aggression, when returned back to the group, if it was treated with isoctocin, an analogue version of oxytocin, versus the treatment with saline [52].

However, there are still a lot of controversial results in this area of research, since for example other groups failed to find any significant effects for the administration of the intranasal oxytocine on aggressive behaviour for example in subjects with antisocial personality disorder. In this way, in a recent study from September 2015 it was found that intransal oxytocine generated little effects in aggression and anyway not related to dose of oxytocin administrated, as judged by a well-validated laboratory task of human aggression called point subtraction aggression paradigm [57].

Even more, it was showed that in non-lacting female rats (so outside the already classical and well known perspective that oxytocin is implicated in defensive maternal aggression $[53,54]$ ) there is surprising potential for an anti-aggressive effect of synthetic oxytocin administration, as determined through an original behavioural approach such as novel female resident-intruder test for spontaneous female aggressive behaviour [58]. In fact, there are previous correlative studies showing a significant correlation between reduced oxytocin concentration in the cerebrospinal fluid of some patients and aggressive behaviour [59], while some authors are strongly believing that the aforementioned intranasal administration of oxytocin is exhibiting pro-social behaviours [60].

There are authors stating that these different effects of oxytocin on aggression, but also on other related superior behaviours, could be explained by the different ways of oxytocin administration (peripheral vs. intranasal), different dosage or by different experimental setup (e.g. looking at outor in-groups members or which is the basic level of aggressive responding in that individual) [50, 61-63].

\section{Conclusions}

Thus, it seems that violent behaviour and impulsive traits present a multifactorial substrate that is determined by genetic and non-genetic factors. In this way, aggressivity is regulated by brain regions such as the amygdala, which controls neural circuits for triggering defensive, aggressive or avoidant behaviour, while the dysfunction of certain neural circuits responsible for emotional control seems to induce violent behaviours. Moreover, besides the amygdala, other brain structures such as the anterior cingulate cortex and prefrontal cortex regions seem to modulate circuits involved in aggressive behaviour. Regarding the genetic aspects, we could also mention the mutations in the monoamine oxidase or the polymorphisms of the genes involved in the metabolism of serotonin, such as tryptophan hydroxylase. Also, besides the low levels of serotonin metabolites which seem to be associated with impulsive and aggressive traits it seems that reduced levels of glutamate, as well as testosterone, vasopressin hypochloesterolemia or oxytocin modifications could be related to the aggressive behaviour.

\section{Acknowledgments}

Padurariu Manuela, Ciobica Alin and Romeo Dobrin are supported by a PN-II-RU-TE-2014-41886 grant called "A complex study regarding the relevance of oxytocin administration in some animal models of neuropsychiatric disorders", number 120 from 01/10/2015.

\section{References}

[1]. C. Anderson, B. Bushman, Human aggression, Annual Review of Psychology. 53 (2000) 2751.

[2]. J. Ramírez, J. Andreu, Aggression, and some related psychological constructs (anger, hostility, and impulsivity); some comments from a research project Neuroscience and Biobehavioural Reviews 30 (2006) 276-91.

[3]. N. Sillmay, Dicţionar de Psihologie Larousse. Ed. Univers Enciclopedic, Bucharest, 1995, 19. 
[4]. M. Briffa, Territoriality and Aggression, Nature Education Knowledge. 3 (2010) 81.

[5]. M. Van Staaden, W. Earcy, R. Hanlon, Signaling Aggression in Aggression Academic Press, Stephen F. Goodwin, 2011.

[6]. D. Maestripieri, D. Functional Aspects of Maternal Aggression in Mammals, Canadian Journal of Zoology. 70 (1992) 1069-1077.

[7]. C. Tanner, Numerical assessment affects aggression and competitive ability: a team-fighting strategy for the ant Formica xerophila, Proceedings. Biological Sciences, 273 (2006) 2737-42.

[8]. A. Rezanur Rahman, Cultural differences in aggression: a case study in Bangladesh, J. Life Earth Sci. 3-4 (2009) 2009.

[9]. M. Bond, Culture and aggression-from context to coercion, Pers Soc Psychol Rev. 8 (2004) 6278.

[10]. C. Cornaggia, M. Beghi, F. Pavone, F. Barale, Aggression in psychiatry wards: a systematic review. Psychiatry Res. 189 (2011) 10-20.

[11]. F. Poulin, M. Boivin, Reactive and Proactive Aggression :Evidence of a Two-Factor Model, Psychological Assessment. 12 (2000) 115-122.

[12]. K. Barlow, B. Grenyer, O. Ilkiw-Lavalle, Prevalence and precipitants of aggression in psychiatric inpatient units, Aust N Z J Psychiatry. 34 (2000) 967-74.

[13]. P. Reebye, Aggression During Early Years - Infancy and Preschool, Child Adolesc Psychiatr Rev. 14 (2005) 16-20.

[14]. H. Heyne, S. Lautenschläger, R. Nelson, F. Besnier, M. Rotival, A. Cagan, R. Kozhemyakina, I. Plyusnina, L. Trut, O. Carlborg, E. Petretto, L. Kruglyak, S. Pääbo, T. Schöneberg, F. Albert, Genetic influences on brain gene expression in rats selected for tameness and aggression, Genetics. 198 (2014) 1277-90.

[15]. M. Bortolato, K. Chen, S. Godar, G. Chen, W. Wu, I. Rebrin I, M. Farrell, A. Scott, C. Wellman, J. Shih, Social deficits and perseverative behaviours, but not overt aggression, in MAO-A hypomorphic mice, Neuropsychopharmacology. 36 (2011) 2674-88.

[16]. A. Marusic, A. Farmer, Genetic risk factors as possible causes of the variation in European suicide rates, Br J Psychiatry. 179 (2001) 194-6.

[17]. A. Takahashi, M. Isabel, R. Quadros, M. de Almeida, K. Miczek, Behavioural and Pharmacogenetics of Aggressive Behaviour, Curr Top Behav Neurosci. 12 (2012) 73-138.

[18]. P. Trzepacz, P. Yu, P. Bhamidipati, B. Willis, T. Forrester, L. Tabas, A. Schwarz, Alzheimer's Disease Neuroimaging Initiative. Frontolimbic atrophy is associated with agitation and aggression in mild cognitive impairment and Alzheimer's disease, Alzheimers Dement. 9 (2013) S95-S104.

[19]. A. New, E. Hazlett, M. Buchsbaum, Blunted prefrontal cortical 18-fluorodeoxyglucose positron emission response to metachloro-phenylpiperazine in impulsive aggression, Arch Gen Psychiatry. 59 (2002) 621-629.

[20]. A. Falkner, P. Dollar, P. Perona, D. Anderson, D. Lin, Decoding ventromedial hypothalamic neural activity during male mouse aggression, J Neurosci. 34 (2014) 5971-84.

[21]. L. van Elst, F. Woermann, L. Lemieux, P. Thompson, M. Trimble, Affective aggression in patients with temporal lobe epilepsy: a quantitative MRI study of the amygdala, Brain. 123 (2000) 234-43. 
[22]. D. Dougherty, F. Moeller, J. Bjork, D. Marsh, Plasma L-tryptophan depletion and aggression, Adv Exp Med Biol. 467 (1999) 57-65.

[23]. G. Pandey, Biological basis of suicide and suicidal behaviour. Bipolar Disord. 15 (2013) 524-41.

[24]. H. Soderstrom, K. Blennow, A. Sjodin, A. Forsman, New evidence for an association between the CSF HVA:5-HIAA ratio and psychopathic traits, J Neurol Neurosurg Psychiatry. 74 (2003) 918-921.

[25]. E. Audero, B. Mlinar, G. Baccini, Z. Skachokova, R. Corradetti, C. Gross, Suppression of serotonin neuron firing increases aggression in mice, J Neurosci. 33 (2013) 8678-88.

[26]. C. Widom, L. Brzustowicz, MAOA and the "cycle of violence": childhood abuse and neglect, MAOA genotype, and risk for violent and antisocial behaviour, Biol Psychiatry. 60 (2006) 684-910.

[27]. O. Cases, I. Seif, J. Grimsby, P. Gaspar, K. Chen, S. Pournin, U. Müller, Aggressive behaviour and altered amounts of brain serotonin and norepinephrine in mice lacking MAOA, Science. 268 (1995) 1763-6.

[28]. A. Caspi, J. McClay, T. Moffitt, J. Mill, J. Martin, I. Craig, A. Taylor, Role of genotype in the cycle of violence in maltreated children, Science. 297 (2002) 851-854.

[29]. J. Kim-Cohen, A. Caspi, A. Taylor, B. Williams, R. Newcombe, I. Craig, T. Moffitt, MAOA, maltreatment, and gene-environment interaction predicting children's mental health: new evidence and a meta-analysis, Mol Psychiatry. (2006)

[30]. N. Alia-Klein, R. Goldstein, A. Kriplani, J. Logan, D. Tomasi, B. Williams, F. Telang, E. Shumay, A. Biegon, I. Craig, F. Henn, G. Wang, N. Volkow, J. Fowler, Brain monoamine oxidase A activity predicts trait aggression, J Neurosci. 28 (2008) 5099-5104.

[31]. M. Niciu, B. Kelmendi, G. Sanacora, Overview of glutamatergic neurotransmission in the nervous system, Pharmacology, Biochemistry, and Behaviour. 100 (2012) 656-64.

[32]. K. Schubert, M. Shaikh, A. Siegel, NMDA receptors in the midbrain periaqueductal gray mediate hypothalamically evoked hissing behaviour in the cat, Brain Research. 726 (1996) 8090 .

[33]. R. Bandler, Identification and midbrain periaqueductal grey neurones mediating aggressive and defensive behaviour by intracerebral microinjections of excitatory amino acids. New York: Alan R. Liss, Inc, (1984) 369-91

[34]. E. Coccaro, R. Lee, P. Vezina, Cerebrospinal fluid glutamate concentration correlates with impulsive aggression in human subjects, J Psychiatr Res. 47 (2013) 1247-53.

[35]. K. Chichinadze, T. Domianidze, T. Matitaishvili, N. Chichinadze, A. Lazarashvili, Possible relation of plasma testosterone level to aggressive behaviour of male prisoners, Bull Exp Biol Med. 149 (2010) 7-9.

[36]. M. Giammanco, G. Tabacchi, S. Giammanco, D. Di Majo, M. La Guardia, Testosterone and aggressiveness, Med Sci Monit. 11 (2005) 136-45.

[37]. P. Negri-Cesi, A. Colciago, F. Celotti, M. Motta, Sexual differentiation of the brain: role of testosterone and its active metabolites, J Endocrinol Invest. 27 (2004) 120-7.

[38]. Y. Kuepper, N. Alexander, R. Osinsky, E. Mueller, A. Schmitz, P. Netter, Aggression-interactions of serotonin and testosterone in healthy men and women, Behav Brain Res. 206 (2010) 93-100. 
[39]. G. Van Wingen, L. Ossewaarde, T. Backstrom, E. Hermans, G. Fernandez, Gonadal hormone regulation of the emotion circuitry in humans, Neuroscience. 191 (2011) 38-45.

[40]. S. Caughey, S. Klampfl, V. Bishop, J. Pfoertsch, I. Neumann, O. Bosch, S. Meddle, Changes in the intensity of maternal aggression and central oxytocin and vasopressin V1a receptors across the peripartum period in the rat, J Neuroendocrinol. 23 (2011) 113-24.

[41]. B. Golomb, T. Kane, J. Dimsdale, Severe irritability associated with statin cholesterollowering drugs, QJM. 97(2004) 229-35.

[42]. C. Ferris, Vasopressin/oxytocin and aggression, Novartis Found Symp. 2005;268:190-8

[43]. C. Widom, L. Brzustowicz, MAOA and the "cycle of violence": childhood abuse and neglect, MAOA genotype, and risk for violent and antisocial behaviour, Biol Psychiatry. 60 (2006) 684-910.

[44]. E. Coccaro, R. Kavoussi, R. Hauger, T. Cooper, C. Ferris, Cerebrospinal fluid vasopressin levels: correlates with aggression and serotonin function inpersonality-disordered subjects, Arch Gen Psychiatry. 55 (1998) 708-14.

[45]. Yatawara CJ, Einfeld SL, Hickie IB, Davenport TA, Guastella AJ. The effect of oxytocin nasal spray on social interaction deficits observed in young children with autism: a randomized clinical crossover trial. Mol Psychiatry. 2015 Oct 27. doi: 10.1038/mp.2015.162

[46]. Guastella AJ, Ward PB, Hickie IB, Shahrestani S, Hodge MA, Scott EM, Langdon R. A single dose of oxytocin nasal spray improves higher-order social cognition in schizophrenia. Schizophr Res. 2015;168(3):628-33.

[47]. Alvares GA, Chen NT, Balleine BW, Hickie IB, Guastella AJ. Oxytocin selectively moderates negative cognitive appraisals in high trait anxious males. Psychoneuroendocrinology. 2012;37(12):2022-31.

[48]. Einfeld SL, Smith E, McGregor IS, Steinbeck K, Taffe J, Rice LJ, Horstead SK, Rogers N, Hodge MA, Guastella AJ. A double-blind randomized controlled trial of oxytocin nasal spray in Prader Willi syndrome. Am J Med Genet A. 2014;164A(9):2232-9.

[49]. Dadds MR, Moul C, Cauchi A, Dobson-Stone C, Hawes DJ, Brennan J et al. Methylation of the oxytocin receptor gene and oxytocin blood levels in the development of psychopathy. Dev Psychopathol. 2014;26(1):33-40.

[50]. Hernádi A, Kis A, Kanizsár O, Tóth K, Miklósi B, Topál J. Intranasally administered oxytocin affects how dogs (Canis familiaris) react to the threatening approach of their owner and an unfamiliar experimenter. Behav Processes. 2015;119:1-5.

[51]. Rault JL, Carter CS, Garner JP, Marchant-Forde JN, Richert BT, Lay DC Jr. Repeated intranasal oxytocin administration in early life dysregulates the HPA axis and alters social behaviour. Physiol Behav. 2013;112-113:40-8.

[52]. Hellmanna J, Reddonb A., Ligockia I., O'Connorb C, Kelly A., Susan E. et al. Group response to social perturbation: impacts of isotocin and the social landscape. Animal Behaviour. 2015;105:55-62.

[53]. Bosch OJ, Meddle SL, Beiderbeck DI, Douglas AJ, Neumann ID. Brain oxytocin correlates with maternal aggression: link to anxiety. J Neurosci. 2005;25(29):6807-15.

[54]. Bosch OJ. Maternal aggression in rodents: brain oxytocin and vasopressin mediate pup defence. Philos Trans R Soc Lond B Biol Sci. 2013;368(1631):20130085. 
[55]. DeWall C, Gillath O., Pressman D., Black L., Bartz A., Moskovitz J., Stetler A. When the Love Hormone Leads to Violence: Oxytocin Increases Intimate Partner Violence Inclinations Among High Trait Aggressive People Social Psychological and Personality Science. February 12, 2015:doi:10.1177/1948550613516876.

[56]. Lind NM, Moustgaard A, Jelsing J, Vajta G, Cumming P, Hansen AK. The use of pigs in neuroscience: modeling brain disorders. Neurosci Biobehav Rev 2007;31:728-51.

[57]. AlcornIII L., Rathnayaka N., Swann C., Gerard Moeller F., Lane D. Effects of Intranasal Oxytocin on Aggressive Responding in Antisocial Personality Disorder. The Psychological Record. 2015;65(4):691-703.

[58]. De Jong D, Beiderbeck D., Neumann I. Oxytocin reduces aggressive behaviour in virgin female rats. Neuroscience 2013 - Society for Neuroscience, Nov 9-13, San Diego, poster no. 754.09 .

[59]. Lee H, Macbeth A, Pagani J, Young W. Oxytocin: the great facilitator of life. Prog. Neurobiol. 2009;88:127-151.

[60]. Romero T, Nagasawa M, Mogi K, Hasegawa T, Kikusui T. Oxytocin promotes social bonding in dogs. Proc. Natl. Acad. Sci. 20104;111:9085-9090.

[61]. Alcorn J, Dias N, Moeller F, Lane S. A preliminary analysis of aggressive behaviour under oxytocin dose. Drug Alcohol Depend. 2014;140:4.

[62]. De Dreu C. Oxytocin modulates cooperation within and competition between groups: an integrative review and research agenda. Horm. Behav. 2012;61:419-428.

[63]. De Dreu CK, Greer LL, Handgraaf MJ, Shalvi S, Van Kleef GA, Baas M, Ten Velden FS, Van Dijk E, Feith SW. The neuropeptide oxytocin regulates parochial altruism in intergroup conflict among humans. Science. 2010;328(5984):1408-11. 